\title{
Fermentation products of Cordyceps militaris enhance performance and modulate immune response of weaned piglets
}

\author{
Y.H. Cheng ${ }^{1 \#}$, C.M. Wen ${ }^{2}$, A. Dybus ${ }^{3}$ \& W.S. Proskura ${ }^{3}$ \\ ${ }^{1}$ Department of Biotechnology and Animal Science, National I-Lan University, I-Lan, Taiwan \\ ${ }^{2}$ Department of Life Sciences, National University of Kaohsiung, Kaohsiung, Taiwan \\ ${ }^{3}$ Laboratory of Molecular Cytogenetics, West Pomeranian University of Technology, Szczecin, Poland
}

(Received 8 October 2015; Accepted 3 March 2016; First published online 7 May 2016)

Copyright resides with the authors in terms of the Creative Commons Attribution 2.5 South African Licence.
See: http://creativecommons. org/licenses/by/2.5/za
Condition of use: The user may copy, distribute, transmit and adapt the work, but must recognise the authors and the South African Journal
of Animal Science.

\begin{abstract}
The aim of this study was to investigate the effect of supplementation of Cordyceps militaris fermentation products (CMF) on growth performance and immunocompetence of piglets. The study involved three groups of animals, which were supplemented with $\operatorname{CMF~}(500,1000$ and $1500 \mu \mathrm{g} / \mathrm{kg}$ feed), and a control group. CMF supplementation significantly increased growth performance in weaned piglets. Bodyweight gain, average daily gain and feed intake in animals supplemented with $1000 \mu \mathrm{g} \mathrm{CMF} / \mathrm{kg}$ feed were significantly higher in comparison with the control group. In addition, CMF supplementation only significantly increased the synthesis of Th1 cytokines, as indicated by the levels of IL-2 and IFN-y. The piglets fed with the CMF supplement displayed an increased cellular immune response. Indeed, alveolar macrophages isolated from piglets supplemented with 1000 and $1500 \mu \mathrm{g} \mathrm{CMF} / \mathrm{kg}$ feed had significantly higher chemotactic and phagocytic indices than those isolated from piglets that received the control feed or feed supplemented with $500 \mu \mathrm{g} \mathrm{CMF} / \mathrm{kg}$. In relation to the absence of effect on Th2 cytokines, the CMF supplement had no effect on hog cholera antibody titre. In summary, feed supplementation with CMF improves growth performance and enhances cell-mediated immunity. CMF supplementation may thus be useful at weaning to counteract physiological and immunological stress during this period.
\end{abstract}

Keywords: Cellular immunity, growth performance, humoral immunity, Th1/Th2 cytokines

\#Corresponding author: yhcheng@niu.edu.tw

\section{Introduction}

Cordyceps militaris is a well-known Chinese medicinal fungus that has been used for a long time as a nutraceutical food in Korea, China and Japan. These entomopathogenic fungi have a wide spectrum of pharmacological activities ( $\mathrm{Ng} \&$ Wang, 2005). It shows antioxidant, anti-inflammatory, antitumor, antiociceptive, antifibrotic and antiangiogenic activities ( $\mathrm{Ng} \&$ Wang, 2005), and immunopotentiation properties (Kuoet al., 2007). C. militaris was reported to improve insulin resistance and insulin secretion (Cheng et al., 2012). This medicinal fungus was used traditionally to cure hyposexuality and hyperlipidemia (Huang et al., 2004; Guo et al., 2010), and to treat asthma and lung inflammation (Wang et al., 2007). In turn, C. militaris is known to demonstrate antiviral activity (Jiang et al., 2011). The medical potential of metabolites of C. militaris has been reviewed and discussed comprehensively in the past (Das et al., 2011; Tuli et al., 2013).

Cordycepin is the major active secondary metabolite of $C$. militaris. This nucleoside analogue displays antimicrobial and antitumor activities (Ahn et al., 2000; Nakamura et al., 2006) and modulates cytokine secretion of human peripheral blood lymphocytes (Zhou et al., 2002). At molecular level, cordycepin regulates the expression of cycloxygenase-2, inducible nitric oxide synthase and TNF- $\alpha$ (Kimet al., 2006). Therefore, when used as a food additive, it may affect the immune system in particular (Das et al., 2011; Tuli et al., 2013). The aim of this study was to evaluate the effects of CMF supplementation in feeds on the growth performance, immunocompetence and cytokine expression of weaned piglets.

\section{Materials and Methods}

The mycelium of C. militaris (BCRC 32219) was grown in a jar fermenter containing $250 \mathrm{~g}$ wheat medium, which contained $0.1 \% \mathrm{CaCO}_{3}, 0.05 \% \mathrm{MgSO}_{4}, 0.1 \% \mathrm{NaH}_{2} \mathrm{PO}_{4}, 50 \% \mathrm{H}_{2} \mathrm{O}, 0.05 \% \mathrm{KH}_{2} \mathrm{PO}_{4}$ and $1 \%$ 
glucose cultivated at $22^{\circ} \mathrm{C}$ for 14 days. The cordycepin content in the fermentative products was assessed with high-performance liquid chromatography (Chang et al., 2005). Briefly, after filtration, samples were subjected to cordycepin analysis via SPD-10A HPLC (Shimadzu, Japan) with a pre-packed LiChrospher 100 RP-18 column (Merck, Darmstadt, Germany). The mobile phase was a mixture of methanol and $0.02 \mathrm{M}$ $\mathrm{KH}_{2} \mathrm{PO}_{4}$ (15:85). Elution was performed at a flow rate of $1 \mathrm{~mL} / \mathrm{min}$ and determined with a variable-wavelength UV detector (10A VP, Shimadzu, Tokyo, Japan) at $254 \mathrm{~nm}$. The cordycepin content of the CMF supplement in this experiment was $5.09 \mathrm{mg} / \mathrm{g}$.

A total of 144 three-week-old cross-bred (Landrace $\times$ Yorkshire $\times$ Duroc) weanling piglets (mean $=6 \mathrm{~kg})$ was randomly allocated to four treatment groups ( $\mathrm{n}=36$ per group): a control group and three groups supplemented with 500, 1000 and $1500 \mu \mathrm{g}$ CMF per kg basal diet. The basal diet is given in Table 1. Water and feed were provided ad libitum throughout the entire experimental period of four weeks. The animal experiments were performed according to the Guide for the Care and Use of Laboratory Animals (National I-Lan University, May/20/2013). Bodyweights were determined at weaning and at the end of the experiment. Feed intake per pen was calculated at weekly intervals. At the end of the experiment, the pigs were killed by electrical stunning, coupled with exsanguination, and blood was collected and centrifuged to harvest serum for biochemistry and antibody measurement. The spleen was cut into $2-3 \mathrm{~mm}$ fragments, snap frozen in liquid nitrogen, and stored at $-80^{\circ} \mathrm{C}$ until the cytokine expression assays were performed.

Table 1 Composition of experimental diets (as-fed basis)

\begin{tabular}{lc}
\hline Ingredients (\%) & Relative proportions \\
\hline Maize & 62.25 \\
Soybean meal (CP 44\%) & 22.50 \\
Fish meal (CP 60\%) & 5.00 \\
Whey & 6.00 \\
Dicalcium phosphate & 1.85 \\
Limestone, pulverized & 1.00 \\
Salt & 0.50 \\
L-Lysine & 0.25 \\
DL-Methionine & 0.07 \\
Choline chloride, 50\% & 0.08 \\
Vitamin premix & 0.30 \\
Trace mineral premix* & 0.20 \\
Calculated nutrient composition (g/kg) & \\
Crude protein & 191.0 \\
Lysine & 11.2 \\
Sulphur amino acid & 7.9 \\
Total phosphorus & 7.4 \\
Calcium & 8.3 \\
Metabolizable energy (MJ/kg) & 13.69 \\
\end{tabular}

\footnotetext{
*Provided per kilogram of diet: vitamin A (retinyl palmitate), $5000 \mathrm{IU}$; cholecalciferol, $500 \mathrm{IU}$; vitamin $\mathrm{E}$ (DL- $\alpha$-tocopheryl acetate), $20 \mathrm{IU}$; vitamin $\mathrm{K}_{3}, 1.25 \mathrm{mg}$; thiamin, $4.2 \mathrm{mg}$; riboflavin, $4.0 \mathrm{mg}$; pantothenic acid, $15.2 \mathrm{mg}$; niacin, $37.3 \mathrm{mg}$; pyridoxine, $6.0 \mathrm{mg}$; choline, $1320 \mathrm{mg}$; folic acid, $1.4 \mathrm{mg}$; biotin, $0.23 \mathrm{mg}$; vitamin $\mathrm{B}_{12}, 15 \mu \mathrm{g}$; ethoxyquin, 120 mg; manganese, 35 mg; zinc, $133 \mathrm{mg}$; iron, 123 mg; copper, $23 \mathrm{mg}$.
}

To evaluate the effects of CMF supplement on specific immune response, pigs were injected subcutaneously with one dose of $2 \mathrm{~mL}$ hog cholera vaccine (Taiwan biopharmaceutical company, Kaoshiung, Taiwan.) at weaning, and received a booster two weeks later. Blood samples were taken on day 28 from 12 randomly selected pigs from each treatment group.

The hog cholera antibody titres were measured with ELISA (IDEXX, Westbrook, Maine, USA) and the 
data were expressed as $\log _{2}$ titre. For blood biochemistry, blood from nine pigs per treatment was collected and centrifuged at $2600 \mathrm{~g}$ for $10 \mathrm{~min}$. The sera were collected to determine creatinine, aspartate glucose, cholesterol and triglycerides concentrations, and aminotransferase (AST, EC 2.6.1.1) and alanine aminotransferase (ALT, EC 2.6.1.2) activity, using an automatic clinical chemistry analyser (Roche Cobas Mira Plus, Roche Diagnostic Systems, Inc., USA).

An extraction of total RNA from spleen tissue was performed using an Ultraspec extraction kit (Biotex, Houston, Texas, USA), followed by reverse transcription of RNA. Primer pairs used for specific amplifications of cDNA of various cytokines have been described previously (Oswald et al., 2001). The PCR products were submitted to electrophoresis in 1\% agarose gel containing ethidium bromide and analysed by real-time image capture for quantitative analysis with an internal control of cyclophilin. Primer sequences and numbers of PCR cycles used for the amplification are summarized in Table 2.

Table 2 Oligonucleotide sequences designed for the detection of various porcine cytokines, with annealing temperatures and number of PCR cycles

\begin{tabular}{llcc}
\hline Gene & Oligonucleotide sequences (5'-3') & Annealing temp. $\left({ }^{\circ} \mathbf{C}\right)$ & No. of cycles \\
\hline \multirow{2}{*}{ IL-2 } & F: GATTTAACAGTTGCTTTGAA & 54 & 45 \\
IFN-ã & R: GTTGAGTAGATGCTTTGACA & 58 \\
F: GAAGAAAGGTCAGCCAAGCGC -10 & R: GCTTGATCACATCCATGCTCC & 54 & 40 \\
IL-4 & F: GCATCCACTTCCCAACCA & 54 \\
R: CTTCCTCATCTTCATCGTCAT & F: TACCAGCAACTTCGTCCAC & 54 & 45 \\
& R: ATCGTCTTTAGCCTTTCCAA & 54 & 26 \\
\hline
\end{tabular}

Alveolar macrophages were collected from the pigs by bronchial alveolar lavage with phosphate-buffered saline. The Boyden chamber technique was applied to measure the chemotaxis function of alveolar macrophages as described by Cheng et al. (2006). Chemotaxis was expressed as a chemotactic index, which was calculated by dividing the value for chemo-attracted macrophages by the value for randomly migrated macrophages in the negative (normal saline) control.

To investigate phagocytic activity, the alveolar macrophage coverslip cultures were incubated for 60 min in complete medium with the addition of $2 \mathrm{~mL}$ Candida albicans suspension $\left(1 \times 10^{7} / \mathrm{mL}\right)$. After that, the coverslips were washed with sterile saline, fixed in methanol and stained with May-Grunwald-Giemsa stain. Phagocytosis was expressed as a phagocytic index, which was calculated by dividing the number of macrophage with a phagocytic activity over the total number of macrophages. The average number of internalized $C$. albicans per macrophage was also determined. These quantifications were done on stained coverslip under a microscope at 1000x magnification. Six pigs in each treatment were analysed. A total of 200 macrophages were scored per coverslip.

The results were expressed as the percentage subjected to logarithmic transformation prior to analysis of variance. Statistical significance among the four groups was determined by one-way analysis of variance. Duncan's new multiple range test was used to evaluate differences between means (SAS Institute, Cary, NC, USA). $P$-values of $<0.05$ were considered statistically significant.

\section{Results}

The effects of supplementation of $C$. militaris fermentation products were first analysed on growth performance of weanling pigs (Table 3). The data showed that the various concentrations of CMF supplement significantly increased total and average bodyweight gains $(P<0.05)$. The bodyweight gain was increased in animals receiving the feed supplemented with 1000 or $1500 \mu \mathrm{g}$ CMF. The feed intake of piglets was also enhanced by $8.3 \%$ and $7.1 \%$ in the animal groups receiving the highest CMF supplementation (1000 and $1500 \mu \mathrm{g} / \mathrm{kg}$ feed, respectively). The feed conversion ratio responded in a quadratic manner. When supplemented with 500 and $1000 \mu \mathrm{g} \mathrm{CMF/kg} \mathrm{feed,} \mathrm{animals} \mathrm{had} \mathrm{significantly} \mathrm{lower} \mathrm{feed} \mathrm{conversion} \mathrm{ratios} \mathrm{than}$ those receiving control feed or feed supplemented with $1500 \mu \mathrm{g} \mathrm{CMF/kg.}$

The blood samples collected at the end of the experiment were used to analyse the effect of CMF supplementation on serum biochemistry. As shown in Table 4, no effect on creatinine and cholesterol levels 
appeared as a result of CMF ingestion. By contrast, consumption of CMF significantly reduced AST and ALT activities and glucose and triglycerides concentrations in serum.

Table 3 Effects of Cordyceps militaris fermentation products (CMF) on growth performance of weanling piglets

\begin{tabular}{lcccc}
\hline \multirow{2}{*}{ Item } & \multicolumn{4}{c}{ Concentration of C. militaris fermentation products $(\boldsymbol{\mu g} / \mathbf{k g}$ feed) } \\
\cline { 2 - 5 } & Control & $\mathbf{5 0 0}$ & $\mathbf{1 0 0 0}$ & $\mathbf{1 5 0 0}$ \\
\hline Bodyweight gain (kg/28 days) & $19.9 \pm 1.1^{\mathrm{a}}$ & $21.2 \pm 0.8$ & $22.5 \pm 1.2^{\mathrm{b}}$ & $21.6 \pm 1.1$ \\
Average daily gain (g/d) & $557.2 \pm 30.8^{\mathrm{a}}$ & $598.5 \pm 33.2^{\mathrm{b}}$ & $655.3 \pm 39.4^{\mathrm{b}}$ & $606.6 \pm 41.3^{\mathrm{b}}$ \\
Feed intake(g/d) & $826.4 \pm 63.8^{\mathrm{a}}$ & $834.2 \pm 68.8^{\mathrm{a}}$ & $901.4 \pm 55.4^{\mathrm{b}}$ & $889.7 \pm 52.3^{\mathrm{b}}$ \\
Feed conversion rate (kg feed/kg gain) & $1.47 \pm 0.12^{\mathrm{a}}$ & $1.38 \pm 0.13^{\mathrm{b}}$ & $1.38 \pm 0.14^{\mathrm{b}}$ & $1.48 \pm 0.13^{\mathrm{a}}$ \\
& & & &
\end{tabular}

Results are expressed as means \pm SD ( $n=36$ pigs per group);

Different superscripts within rows indicate statistically significant differences $(P<0.05)$.

Table 4 Effects of Cordyceps militaris fermentation products (CMF) on blood biochemical determinations of weanling piglets

\begin{tabular}{lccrr}
\hline \multirow{2}{*}{ Item } & \multicolumn{2}{c}{ Concentration of $\boldsymbol{C}$. militaris fermentation products $(\boldsymbol{\mu g} / \mathbf{k g}$ feed) } \\
\cline { 2 - 5 } & \multicolumn{1}{c}{ Control } & $\mathbf{5 0 0}$ & $\mathbf{1 0 0 0}$ & $\mathbf{1 5 0 0}$ \\
\hline Creatinine $(\mathrm{mg} / \mathrm{dL})$ & $0.70 \pm 0.10$ & $0.70 \pm 0.00$ & $0.85 \pm 0.05$ & $0.70 \pm 0.14$ \\
Aspartate aminotransferase (U/L) & $101.33 \pm 32.40^{\mathrm{a}}$ & $67.67 \pm 6.12^{\mathrm{b}}$ & $68.00 \pm 3.00^{\mathrm{b}}$ & $63.00 \pm 7.12^{\mathrm{b}}$ \\
Alanine aminotransferase (U/L) & $76.00 \pm 7.27^{\mathrm{a}}$ & $68.67 \pm 17.91^{\mathrm{b}}$ & $65.60 \pm 5.50^{\mathrm{b}}$ & $59.00 \pm 11.52^{\mathrm{b}}$ \\
Glucose (mg/dL) & $116.33 \pm 4.64^{\mathrm{a}}$ & $115.00 \pm 3.26^{\mathrm{a}}$ & $104.50 \pm 1.50^{\mathrm{b}}$ & $108.67 \pm 4.45^{\mathrm{b}}$ \\
Cholesterol (mg/dL) & $72.33 \pm 1.24$ & $76.33 \pm 1.89$ & $74.50 \pm 4.50$ & $78.33 \pm 1.92$ \\
Triglycerides (mg/dL) & $77.00 \pm 10.98^{\mathrm{a}}$ & $71.67 \pm 18.1^{\mathrm{a}}$ & $48.00 \pm 1.00^{\mathrm{b}}$ & $52.67 \pm 13.27^{\mathrm{b}}$
\end{tabular}

Results are expressed as means \pm SD ( $n=9$ randomly selected pigs per group);

Different superscripts within rows indicate statistically significant differences $(P<0.05)$.

The ability of CMF to modulate the cytokine expression at transcriptional level was then investigated on spleen samples from six randomly selected pigs per group. The synthesis of Th1 cytokines (IL-2 and IFN- $\mathrm{y}$ ) and Th2 cytokines (IL-4 and IL-10) was measured by RT-PCR. The mRNA synthesis of Th2 cytokines was modified slightly by supplementation with CMF (Figure 1). By contrast, ingestion of diet supplemented with CMF significantly altered the synthesis of the mRNA encoding for Th1 cytokines in the spleen. For example, the expressions of IL-2 and IFN-y were increased 3.9 and 5.0 fold, respectively, in the spleen of pigs from the $1500 \mu \mathrm{g} / \mathrm{kg}$ CMF supplemented group compared with the spleen of pigs from the control group (Figure 1).

Spleen samples were collected after euthanasia and assayed for expression of IL-2, IFN-Y, IL-4 and IL-10 by RT-PCR. Quantification of the relative cytokine mRNA levels for each sample is expressed in arbitrary units (AU) as the ratio between the cytokine specific RT-PCR product and the corresponding cyclophilin band intensity (mean \pm SEM of six animals per group). Statistical analysis was performed to compare cytokine mRNA expression in the control and CMF-treated animals. Different letters above the bars indicate statistically significant differences between the groups $(P<0.05)$.

Because of the effect of CMF on the Th1/Th2 cytokine balance, it was of interest to investigate the consequence of the ingestion of the CMF supplemented diet on cellular and humoral immune response. Therefore, the functional profile of alveolar macrophages isolated from piglets fed the various diets was determined. As indicated in Table 5, CMF supplementation enhanced the chemotaxis and the phagocytosis of macrophages. Macrophages from animals receiving the diet supplemented with 1000 and $1500 \mu \mathrm{g} \mathrm{CMF} / \mathrm{kg}$ feed demonstrated that the chemotactic indices increased by 2.1 and 2.2 fold, respectively, when compared 
with macrophages from the control animals. Similarly, the phagocytic indices of macrophages obtained from animals receiving the diet supplemented with 1000 and $1500 \mu \mathrm{g} \mathrm{CMF/kg}$ feed were 1.4- and 1.5-fold higher than those observed in animals receiving the control feed. By contrast, CMF supplementation did not modify the number engulfed of $C$. albicans per macrophage.
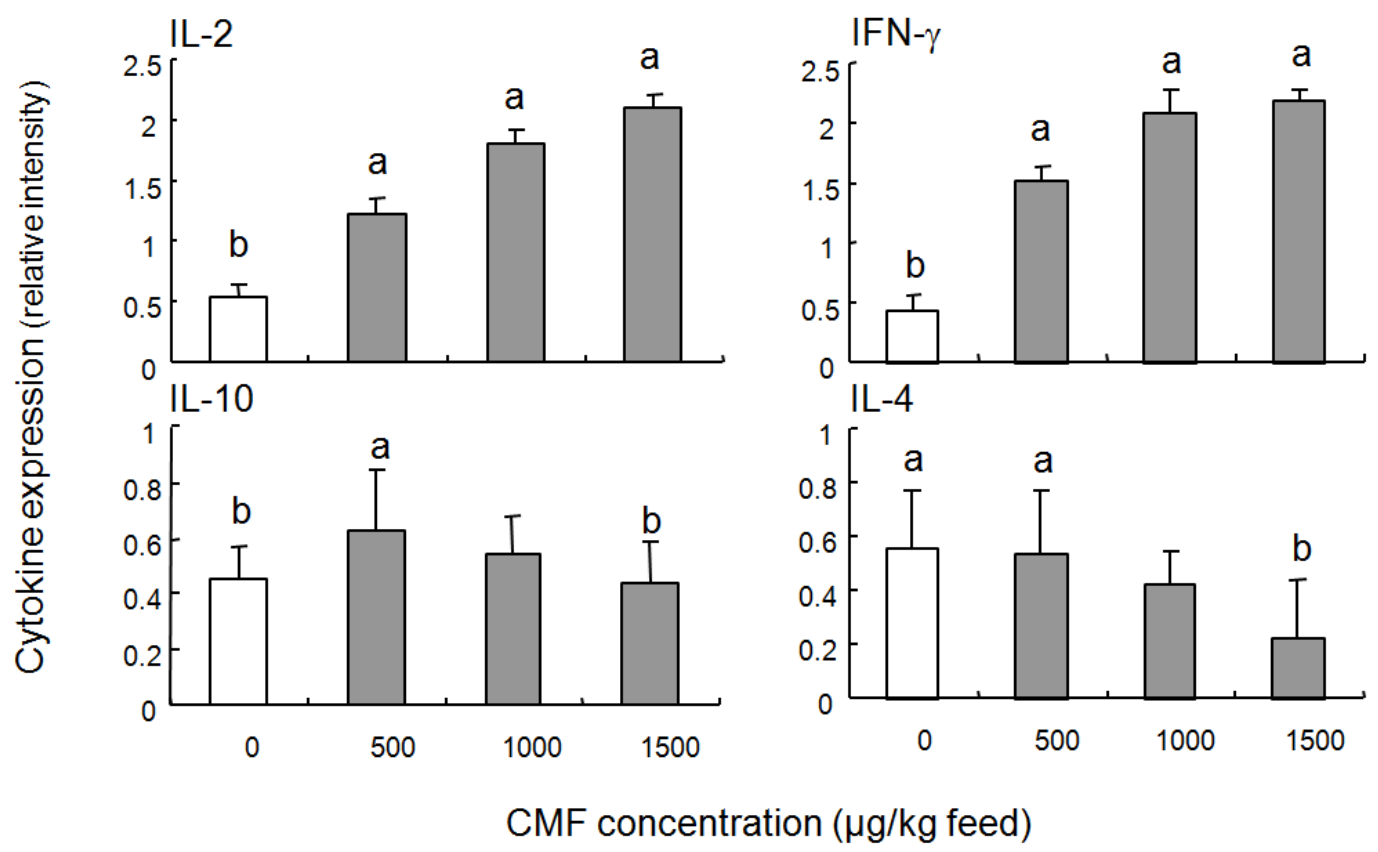

Figure 1 Effect of Cordyceps militaris fermentation products (CMF) on the splenic expression of cytokines in weaning piglets. IL-2: interleukin-2; IL-10: interleukin-10; IL-4: interleukin-4; IFN-ү: interferon-ү. Different letters within histogram indicate statistically significant differences $(P<0.05)$.

Table 5 Effects of Cordyceps militaris fermentation products (CMF) on the functional profile of alveolar macrophages in weanling piglets

\begin{tabular}{lcccc}
\hline \multirow{2}{*}{ Item } & \multicolumn{4}{c}{ Concentration of $\boldsymbol{C}$. militaris fermentation products $(\boldsymbol{\mu g} / \mathbf{k g}$ feed) } \\
\cline { 2 - 5 } & \multicolumn{1}{c}{ Control } & 500 & $\mathbf{1 0 0 0}$ & $\mathbf{1 5 0 0}$ \\
\hline Chemotactic index & $3.13 \pm 0.52^{\mathrm{a}}$ & $3.14 \pm 0.74^{\mathrm{a}}$ & $6.55 \pm 0.48^{\mathrm{b}}$ & $6.81 \pm 0.32^{\mathrm{b}}$ \\
Phagocytic index & $25.9 \pm 2.43^{\mathrm{a}}$ & $24.8 \pm 1.24^{\mathrm{a}}$ & $35.6 \pm 3.91^{\mathrm{b}}$ & $38.2 \pm 3.45^{\mathrm{b}}$ \\
Number of $C$. albicans per macrophage & $1.52 \pm 0.53$ & $1.45 \pm 0.59$ & $1.43 \pm 0.53$ & $1.48 \pm 0.69$
\end{tabular}

Results are expressed as means \pm SD ( $n=6$ randomly selected pigs per group group);

Different superscripts within rows indicate statistically significant differences $(P<0.05)$.

To investigate the influence of CMF on the humoral immune response, the control and the CMF-treated piglets were immunized with a commercial hog cholera vaccine. Antibody levels were measured with ELISA after the second injection in 12 animals per treatment. The data indicate no significant difference among animals receiving the treatments. The antibody titres, expressed as $\log _{2}$, were $25.2 \pm 14.2,25.1 \pm 13.8,26.4 \pm$ 10.2 and $24.8 \pm 11.3$ in animals from the control group and the groups receiving feed supplemented with 500 , 1000 , and $1500 \mu \mathrm{g} \mathrm{CMF/kg}$, respectively.

\section{Discussion}

A cordycepin produced by C. militaris during fermentation has high potential as an immune modulator in the animal feed industry. In the current study it was demonstrated that the supplementation of CMF enhances growth performance and increases the cell-mediated immune response of pigs.

In the present experiment, the authors used a crude fermentative extract of $C$. militaris containing a 
high amount of cordycepin. Cordycepin is not commercially available, and the authors anticipate that purification of this compound would be too expensive for it to be used as a feed additive. Using similar extract, Lin et al. (2007) observed that CMF improved the sperm production in sub-fertile boars. In the current study, the data demonstrated that CMF increases bodyweight gain of piglets and decreases their feed conversion ratio. The underlying mechanism has not been elucidated, but several hypotheses can be proposed. Firstly, CMF may have an anabolic activity. Indeed, Cordyceps sp. has been shown to significantly increase serum testosterone levels in mice (Huang et al., 2004). Secondly, cordyceps extract may act on microbial flora. Previous studies have reported that supplementation of chickens with cordyceps extract resulted in a decrease in the populations of Salmonella sp. and E. coli, and an increase in Lactobacillus sp. in the small intestine (Koh et al., 2003). CMF also demonstrated a potent growth-inhibiting activity toward some pathogenic intestinal bacteria such as Clostridium spp., without adverse effects on the growth of Bifidobacteria and Lactobacilli, suggesting that cordycepin is a selective growth inhibitor of luminal microflora (Ahn et al., 2000). Third, the immuno-stimulatory effect of CMF may account at least partially for the increased performance of the animals. Indeed, domestic animals selected for higher immune response display better zootechnical performance (Wilkie \& Mallard, 1999).

A biochemical analysis of the piglet sera demonstrated that CMF extract was not harmful to the animals. The low blood glucose and triglycerides levels observed in piglets receiving the feed supplemented with the highest dose of CMF suggest that CMF might be beneficial to humans that suffer from hyperglycaemia or hypertriglycemia, as suggested by other studies (Lo et al., 2006).

The main outcome of this study is that CMF acts on the immune system, especially on the Th1/Th2 balance. CD4+ T cells are broadly divided into two subsets, based on the cytokines they secrete, namely Th1 (IL-2 and IFN- $\gamma$ ) and Th2 (IL-4 and IL-10) (Zhou et al., 2009). The development of these T cell subsets is particularly relevant in response to the development of protective immunity to many pathogens (Williams et al., 1995). In the current study the authors observed that the different doses of CMF increased expression of IL-2 and IFN- $\mathrm{Y}$ (Th1 cytokines). Th1 cytokines are implicated in the development of the cellular immune response and macrophage activation (Williams et al., 1995; Ma et al., 2003). The authors therefore postulated that the increase of IL-2 and IFN- $\gamma$ induced by the CMF supplement may increase the cellular immune response. To validate this hypothesis, the chemotaxis and phagocythosis of macrophages were measured. A prolonged exposure (28 days) to feed supplemented with 1000 or $1500 \mu \mathrm{g} \mathrm{CMF} / \mathrm{kg}$ induced a significant increase of the ability of alveolar macrophages to phagocyte Candida albicans.

In agreement with the present study, increased phagocytosis and $\mathrm{H}_{2} \mathrm{O}_{2}$ production was observed in macrophages isolated from mice fed a fermentative extract of another species of Cordyceps (Kuoet al., 2007). In this experiment, the increased cellular response was also associated with an increased expression of Th1 cytokines as measured by the elevated levels of IL-1, IL-6, IL-10 and TNF- $\alpha$. An increased production of inflammatory cytokines was also observed in mice fed with various preparations of $C$. militaris and $C$. sinensis (Sheng et al., 2010; Shin et al., 2010) and in cell lines exposed to these extracts (Han et al., 2010; Meng et al., 2014). Macrophages are key participants in the innate immune system's ability to respond to the invasion of pathogenic organisms. It has been observed that another medicinal fungi, Ganoderma lucidum, was able to increase macrophage chemotaxis ability in pigs (Chen et al., 2008).

This study showed that the CMF supplementation does not modulate antibody titres. These results are correlated with the moderate effect of CMF on the expression of Th2 cytokines. Two separate studies have demonstrated that mycelial extract of $C$. shecocephala and water extract $C$. miltensis, decrease the Th2 response in mice. In these studies, the decreased synthesis of IL4, IL-10 and IL-13 was associated with anti-asthmatic activity (Heo et al., 2010) and a decrease in IgE synthesis (Park et al., 2008). In contrast, Koh et al. (2003), using a hot-water extract of $C$. sinensis, observed increased antibody titre to Newcastle Disease virus in broiler chicks. The fungal strain, the type of extract and the animal species may explain the different results obtained in the various studies.

\section{Conclusions}

Feed supplementation with products of fermentation of Cordyceps militaris improves growth performance of piglets significantly, and enhances cell-mediated immunity. CMF supplementation, at least at $1000 \mu \mathrm{g} / \mathrm{kg}$, may thus be useful at weaning to counteract physiological and immunological stress during this period.

\section{Acknowledgements}

This research was partly supported by Council of Agriculture, Taiwan, Republic of China [96 Agri-2.1.3-Mu-U1(3)]. We would like to thank Isabelle Oswald from Toulouse/INRA/France for her suggestion and valuable criticism on the manuscript. 


\section{Authors' contributions}

CMW was in charge of organizing and supervising the course of the project and the article. AD and WSP took responsibility of the logical interpretation and presentation of the results. YHC contributed to the whole research proposal, project application and writing of the manuscript.

\section{Conflict of interest declaration}

No conflicts of interest are declared by the authors.

\section{References}

Ahn, Y.J., Park, S.J., Lee, S.G., Shin, S.C. \& Choi, D.H., 2000. Cordycepin: selective growth inhibitor derived from liquid culture of Cordyceps militaris against Clostridium spp. J. Agri. Food Chem. 48, 2744-2748.

Chen, S.D., Hsieh, M.C., Chiou, M.T., Lai, Y.S. \& Cheng, Y.H., 2008. Effects of Ganoderma lucidum fermentatives on growth performance and immunocompetences from PCV2 challenge in weanling pigs. Arch. Anim. Nutr. 62, 22-32.

Chang, C.Y., Lue, M.Y. \& Pan, T.M., 2005. Determination of adenosine, cordycepin and ergosterol contents in cultivated Antrodia camphorata by HPLC method. J. Food Drug Anal. 13, 338-342.

Cheng, Y.H., Weng, C.F., Chen, B.J. \& Chang, M.H., 2006. Toxicity of different Fusarium mycotoxins on growth performance, immune responses and efficacy of a mycotoxin degrading enzyme in pigs. Anim. Res. 55, 579-590.

Cheng, Y.W., Chen, Y.I., Tzeng, C.Y., Chen, H.C., Tsai, C.C., Lee, Y.C., Lin, J.G., Lai, Y.K. \& Chang, S.L., 2012. Extracts of Cordyceps militaris lower blood glucose via the stimulation of cholinergic activation and insulin secretion in normal rats. Phytother. Res. 26, 1173-1177.

Das, S.K., Masuda, M., Sakurai, A. \& Sakakibara, M., 2010. Medicinal uses of the mushroom Cordyceps militaris: Current state and prospects. Fitoterapia 81, 961-968.

Guo, P., Kai, Q., Gao, J., Lian, Z.Q., Wu, C.M., Wu, C.A. \& Zhu, H.B., 2010. Cordycepin prevents hyperlipidemia in hamsters fed a high-fat diet via activation of AMP-activated protein kinase. J. Pharmacol. Sci. 113, 395-403.

Han, J.Y., Im, J., Choi, J.N., Lee, C.H., Park, H.J., Park, D.K., Yun, C.H. \& Han, S.H., 2010. Induction of IL-8 expression by Cordyceps militaris grown on germinated soybeans through lipid rafts formation and signalling pathways via ERK and JNK in A549 cells. J. Ethnopharmacol. 127, 55-61.

Heo, J.C., Nam, S.H., Nam, D.Y., Kim, J.G., Lee, K.G., Yeo, J.H., Yoon, C.S., Park, C.H. \& Lee, S.H., 2010. Anti-asthmatic activities in mycelial extract and culture filtrate of Cordyceps sphecocephala J 201. Int. J. Mol. Med. 26, 351-356.

Huang, Y.L., Leu, S.F., Liu, B.C., Sheu, C.C. \& Huang, B.M., 2004. In vivo stimulatory effect of Cordyceps sinensis mycelium and its fractions on reproductive functions in male mouse. Life Sci. 75, 1051-1062.

Jiang, Y., Wong, J.H., Fu, M., Ng, T.B., Liu, Z.K., Wang, C.R., Li, N., Qiao, W.T., Wen, T.Y. \& Liu, F., 2011. Isolation of adenosine, iso-sinensetin and dimethylguanosine with antioxidant and HIV-1 protease inhibiting activities from fruiting bodies of Cordyceps militaris. Phytomedicine 18, 189-193.

Kim, H.G., Shrestha, B., Lim, S.Y., Yoon, D.H., Chang, W.C., Shin, D.J., Han, S.K., Park, S.M., Park, J.H., Park, H.I., Sung, J.M., Jang, Y., Chung, N., Hwang, K.C. \& Kim, T.W., 2006. Cordycepin inhibits lipopolysaccharide-induced inflammation by the suppression of NF-kappaB through Akt and p38 inhibition in RAW 2647 macrophage cells. Eur. J. Pharmacol. 545, 192-199.

Koh, J.H., Suh, H.J. \& Tae, A.S., 2003. Hot-water extract from mycelia of Cordyceps sinensis as a substitute for antibiotic growth promoters. Biotechnol. Lett. 25, 585-590.

Kuo, M.C., Chang, C.Y., Cheng, T.L. \& Wu, M.J., 2007. Immunomodulatory effect of exo-polysaccharides from submerged cultured Cordyceps sinensis: enhancement of cytokine synthesis, CD11b expression, and phagocytosis. Appl. Microbiol. Biotechnol. 75, 769-775.

Lin, W.H., Tsai, M.T., Chen, Y.S., Lai, M.N. \& Jeng, K.C., 2007. Improvement of sperm production in subfertile boars by Cordyceps militaris supplement. Am. J. Chin. Med. 35, 631-641.

Lo, H.C., Hsu, T.H., Tu, S.T. \& Lin, K.C., 2006. Anti-hyperglycemic activity of natural and fermented Cordyceps sinensis in rats with diabetes induced by nicotinamide and streptozotocin. Am. J. Chin. Med. 34, 819-832.

Ma, J., Chen, T., Mandelin, J., Ceponis, A., Miller, N.E., Hukkanen, M., Ma, G.F. \& Konttinen, Y.T., 2003. Regulation of macrophage activation. Cell. Mol. Life Sci. 60, 2334-2346.

Meng, L.Z., Feng, K., Wang, L.Y., Cheong, K.L., Nie, H., Zhao, J. \& Li, S.P., 2014. Activation of mouse macrophages and dendritic cells induced by polysaccharides from a novel Cordyceps sinensis fungus UM01. J. Funct. Foods 9, 242-253.

Nakamura, K., Yoshikawa, N., Yamaguchi, Y., Kagota, S., Shinozuka, K. \& Kunitomo, M., 2006. Antitumor effect of cordycepin (3'-deoxyadenosine) on mouse melanoma and lung carcinoma cells involves adenosine A3 receptor stimulation. Anticancer Res. 26, 43-47.

Ng, T.B. \& Wang, H.X., 2005. Pharmacological actions of Cordyceps, a prized folk medicine. J. Pharmacol. 57, 1509-1519.

Oswald, I.P., Dozois, C.M., Barlagne, R., Fournout, S., Johansen, M.V. \& Bøgh, H., 2001. Cytokine mRNA expression in pigs infected with Schistosoma japonicum. Parasitology 122 (Pt 3), 299-307.

Park, D.K., Choi, W.S., Park, P.J., Kim, E.K., Jeong, Y.J., Choi, S.Y., Yamada, K., Kim, J.D. \& Lim, B.O., 2008. Immunoglobulin and cytokine production from mesenteric lymph node lymphocytes is regulated by extracts of Cordyceps sinensis in C57BI/6N mice. J. Med. Food 11, 784-788.

Sheng, L., Chen, J., Li, J. \& Zhang, W., 2010. An exopolysaccharide from cultivated Cordyceps sinensis and its effects on cytokine expressions of immunocytes. Appl. Biochem. Biotechnol.163, 669-678.

Shin, S., Kwon, J., Lee, S., Kong, H., Lee, S., Lee, C.K., Cho, K., Ha, N.J. \& Kim, K., 2010. Immunostimulatory effects of 
Cordyceps militaris on macrophages through the enhanced production of cytokines via the activation of NF-kappaB. Immune Netw. 10, 55-63.

Tuli, H.S., Sharma, A.K., Sandhu, S.S. \& Kashyap, D., 2013. Cordycepin: a bioactive metabolite with therapeutic potential. Life Sci. 93, 863-869.

Wang, N.Q., Jiang, L.D., Zhang, X.M. \& Li, Z.X., 2007. Effect of dongchong xiacao capsule on airway inflammation of asthmatic patients. Zhongguo Zhong Yao Za Zhi 32,1566-1568.

Wilkie, B. \& Mallard, B., 1999. Selection for high immune response: an alternative approach to animal health maintenance? Vet. Immunol. Immunopathol. 72, 231-235.

Williams, M.A., Caspar, P., Oswald, I.P., Sharma, H.K., Pankewycz, O., Sher, A. \& James, S.L., 1995. Vaccination routes that fail to elicit protective immunity against Schistosoma mansoni induce the production of TGF-beta which down-regulates macrophage antiparasitic activity. J. Immunol. 154, 4693-4700.

Zhou, X., Meyer, C.U., Schmidtke, P. \& Zepp, F., 2002. Effect of cordycepin on interleukin-10 production of human peripheral blood mononuclear cells. Eur. J. Pharmacol. 453, 309-317.

Zhou, L., Chong, M.M. \& Littman, D.R., 2009. Plasticity of CD4+ T cell lineage differentiation. Immunity 30, 646-655. 